\title{
Context Comparison as a Minimum Cost Flow Problem
}

\author{
Vivian Tsang and Suzanne Stevenson \\ Department of Computer Science \\ University of Toronto \\ Canada \\ \{vyctsang, suzanne\}@cs.utoronto.ca
}

\begin{abstract}
Comparing word contexts is a key component of many NLP tasks, but rarely is it used in conjunction with additional ontological knowledge. One problem is that the amount of overhead required can be high. In this paper, we provide a graphical method which easily combines an ontology with contextual information. We take advantage of the intrinsic graphical structure of an ontology for representing a context. In addition, we turn the ontology into a metric space, such that subgraphs within it, which represent contexts, can be compared. We develop two variants of our graphical method for comparing contexts. Our analysis indicates that our method performs the comparison efficiently and offers a competitive alternative to non-graphical methods.
\end{abstract}

\section{Introduction}

Many natural language problems can be cast as a problem of comparing "contexts" (units of text). For example, the local context of a word can be used to resolve its ambiguity (e.g., Schütze, 1998), assuming that words used in similar contexts are closely related semantically (Miller and Charles, 1991). Extending the meaning of context, the content of a document may reveal which document class(es) it belongs to (e.g., $\mathrm{Xu}$ et al., 2003). In any application, once a sensible view of context is formulated, the next step is to choose a representation that makes comparisons possible. For example, in word sense disambiguation, a context of an ambiguous instance can be represented as a vector of the frequencies of words surrounding it. Until recently, the dominant approach has been a non-graphical onecontext comparison is reduced to a task of measuring distributional distance between context vectors. The difference in the frequency characteristics of contexts is used as an indicator of the semantic distance between them.

We present a graphical alternative that combines both distributional and ontological knowledge. We begin with the use of a different context representation that allows easy incorporation of ontological information. Treating an ontology as a network, we can represent a context as a set of nodes in the network (i.e., concepts in the ontology), each with a weight (i.e., frequency). To contrast our work with that of Navigli and Velardi (2005) and Mihalcea (2006), the goal is not merely to provide a graphical representation for a context in which the relevant concepts are connected. Rather, contexts are treated as weighted subgraphs within a larger graph in which they are connected via a set of paths. By incorporating the semantic distance between individual concepts, the graph (representing the ontology) becomes a metric space in which we can measure the distance between subgraphs (representing the contexts to be compared).

More specifically, measuring the distance between two contexts can be viewed as solving a minimum cost flow (MCF) problem by calculating the amount of "effort" required for transporting the flow from one context to the other. Our method has the advantage of including semantic information (by making use of the graphical structure of an ontology) without losing distributional information (by 
using the concept frequencies derived from corpus data).

This network flow formulation, though supporting the inclusion of an ontology in context comparison, is not flexible enough. The problem is rooted in the choice of concept-to-concept distance (i.e., the distance between two concepts, to contrast it from the overall semantic distance between two contexts). Certain concept-to-concept distances may result in a difficult-to-process network which severely compromises efficiency. To remedy this, we propose a novel network transformation method for constructing a pared-down network which mimics the structure of the more precise network, but without the expensive processing or any significant information loss as a result of the transformation.

In the remainder of this paper, we first present the underlying network flow framework, and develop a more efficient variant of it. We then evaluate the robustness of our methods on a context comparison task. Finally, we conclude with an analysis and some future directions.

\section{The Network Flow Method}

\subsection{Minimum Cost Flow}

As a standard example of an MCF problem, consider the graphical representation of a route map for delivering fresh produce from grocers (supply nodes) to homes (demand nodes). The remaining nodes (e.g., intersections, gas stations) have neither a supply nor a demand. Assuming there are sufficient supplies, the optimal solution is to find the cheapest set of routes from grocers to homes such that all demands are satisfied.

Mathematically, let $G=(N, E)$ be a connected network, where $N$ is the set of nodes, and $E$ is the set of edges. ${ }^{1}$ Each edge has a cost $c: E \rightarrow \mathbb{R}$, which is the distance of the edge. Each node $i \in N$ is associated with a value $b(i)$ such that $b: N \rightarrow \mathbb{R}$ indicates its available supply $(b(i)>0)$, its demand $(b(i)<0)$, or neither $(b(i)=0)$. The goal is to find a solution for each node $i$ such that all the flow passing through $i$ satisfies its supply or demand requirement $(b(i))$. The flow passing through node $i$ is captured by $x: E \rightarrow \mathbb{R}$ such that we can observe the com-

\footnotetext{
${ }^{1}$ Most ontologies are hierarchical, thus, in the case of a forest, adding an arbitrary root node yields a connected graph.
}

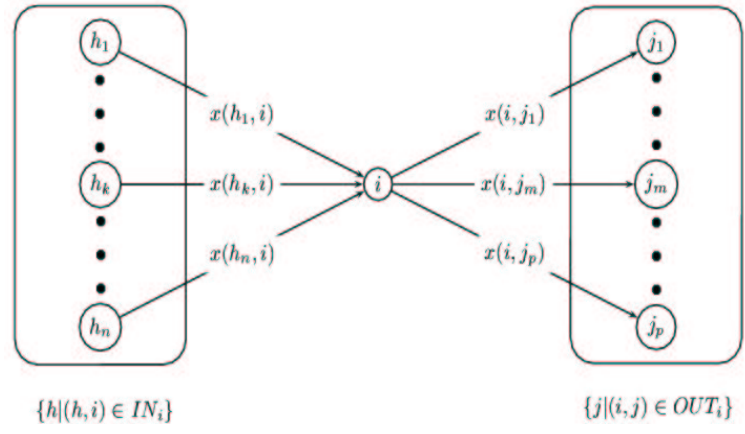

Figure 1: An illustration of flow entering and exiting node $i$.

bined incoming flow, $\sum_{(h, i) \in I N_{i}} x(h, i)$, from the entering edges $I N_{i}$, as well as the combined outgoing flow, $\sum_{(i, j) \in O U T_{i}} x(i, j)$, via the exiting edges $O U T_{i}$. (See Figure 1.) If a feasible solution can be found, the net flow (the difference between the entering and exiting flow) at each node must fulfill the corresponding supply or demand requirement.

Formally, the MCF problem can be stated as:

Minimize

$$
z(\vec{x})=\sum_{(i, j) \in E} c(i, j) \cdot x(i, j)
$$

subject to

$$
\begin{gathered}
\sum_{(i, j) \in O U T_{i}} x(i, j)-\sum_{(h, i) \in I N_{i}} x(h, i)=b(i), \forall i \in N \\
x(i, j) \geq 0, \forall(i, j) \in E
\end{gathered}
$$

The constraint specified by (2) ensures that the difference between the flow entering and exiting each node $i$ matches its supply or demand $b(i)$ exactly. The next constraint (3) ensures that the flow is transported from the supply to the demand but not in the opposite direction. Finally, selecting route $(i, j)$ requires a transportation "effort" of $c(i, j)$ (cost of the route) multiplied by the amount of supply transported $x(i, j)$ (the term inside the summation in eqn. (1)). Taking the summation of the effort, $c(i, j) \cdot x(i, j)$, of cheapest routes yields the desired distance between the supply and the demand.

\subsection{Semantic Distance as MCF}

To cast our context comparison task into this framework, we first represent each context as a vector of concept frequencies (or a context profile for the remainder of this paper). The profile of one context is chosen as the supply and the other as the demand. The concept frequencies of the profiles are normalized, so that the total supply always equals the total 
demand. The cost of the routes between nodes is determined by a semantic distance measure defined over any two nodes in the ontology. Now, as in the grocery delivery domain, the goal is to find the $\mathrm{MCF}$ from supply to demand.

We can treat any ontology as the transport network. A relation (such as hyponymy) between two concepts $i$ and $j$ is represented by an edge $(i, j)$, and the cost $c$ on each edge can be defined as the semantic distance between the two concepts. This semantic distance can be as simple as the number of edges separating the concepts, or more sophisticated, such as Lin's (1998) information-theoretic measure. (See Budanitsky and Hirst (2006) for a survey of such measures).

Numerous methods are possible for converting the word frequency vector of a context to a concept frequency vector (i.e., a context profile). One simple method is to transfer each element in the word vector (i.e., the frequency of each word) to the corresponding concepts in the ontology, resulting in a vector of concept frequencies. In this paper, we have chosen a uniform distribution of word frequency counts among concepts, instead of a weighted distribution towards the relevant concepts for a particular text. Since we wish to evaluate the strength of our method alone without any additional NLP effort, we bypass the issue of approximating the true distribution of the concepts via word sense disambiguation or classbased approximation methods, such as those by Li and Abe (1998) and Clark and Weir (2002).

To calculate the distance between two profiles, we need to cast one profile as the supply $(S)$ and the other as the demand $(D)$. Note that our distance is symmetric, so the choice of the supply and the demand is arbitrary. Next, we must determine the value of $b(i)$ at each concept node $i$; this is just the difference between the (normalized) supply frequency $f_{S}(i)$ and demand frequency $f_{D}(i)$ :

$$
b(i)=f_{S}(i)-f_{D}(i)
$$

This formula yields the net supply/demand, $b(i)$, at node $i$. Recall that our goal is to transport all the supply to meet the demand-the final step is to determine the cheapest routes between $S$ and $D$ such that the constraints in (2) and (3) are satisfied. The total distance of the routes, or the MCF, $z(\vec{x})$ in eqn. (1), is the distance between the two context profiles.
Finally, it is important to note that the MCF formulation does not simply find the shortest paths from the concept nodes in the supply to those in the demand. Because a profile is a frequency-weighted concept vector, some concept nodes are weighted more heavily than others, and the routes between such nodes across the two profiles are also weighted more heavily. Indeed, in eqn. (1), the cost of each route, $c(i, j)$, is weighted by $x(i, j)$ (how much supply, or frequency weight, is transported between nodes $i$ and $j$ ).

\section{Graphical Issues}

As alluded to in the introduction, certain conceptto-concept distances pose a problem to solving the MCF problem easily. The details are described next.

\subsection{Additivity}

In theory, our method has the flexibility to incorporate different concept-to-concept distances. The issue lies in the algorithms for solving MCF problems. Existing algorithms are greedy-they take a stepwise "localist" approach on the set of edges connecting the supply and the demand; i.e., at each node, the cheapest outgoing edge is selected. The assumption is that the concept-to-concept distance function is additive. Mathematically, for any path from node $i$ to node $k,\left\{\left(j_{0}, j_{1}\right), \ldots,\left(j_{n-1}, j_{n}\right)\right\}$, where $i=j_{0}$ and $k=j_{n}$, the distance between nodes $i$ and $k$ is the sum of the distance of the edges along the path:

$$
\operatorname{dist}(i, k)=\sum_{m=0}^{n-1} \operatorname{dist}\left(j_{m}, j_{m+1}\right)
$$

The additivity of a concept-to-concept distance entails that selecting the cheapest edge at each step (i.e., locally) yields the overall cheapest set of routes (i.e., globally). Note that some of the most successful concept-to-concept distances proposed in the CL literature are non-additive (e.g., Lin, 1998; Resnik, 1995). This poses a problem in solving our network flow problem - the global distance between any concepts, $i$ and $k$, cannot be correctly determined by the greedy method.

\subsection{Constructing an Equivalent Bipartite Network}

The issue of non-additive distances can be addressed in the following way. We map the relevant portion 


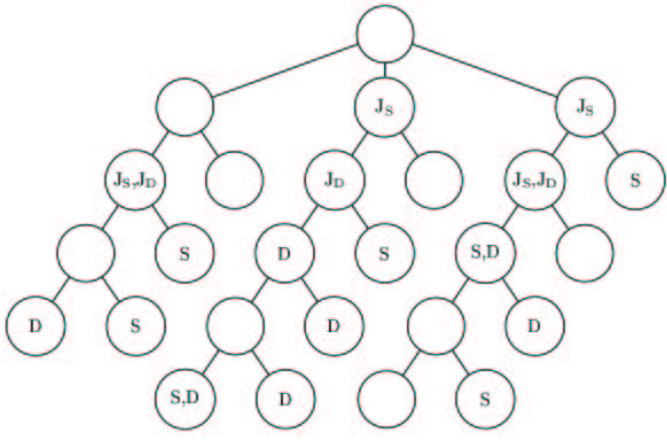

(a)

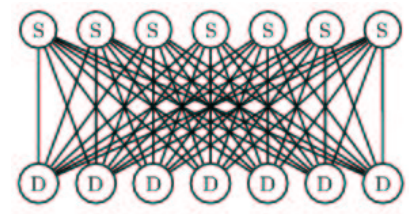

(b)

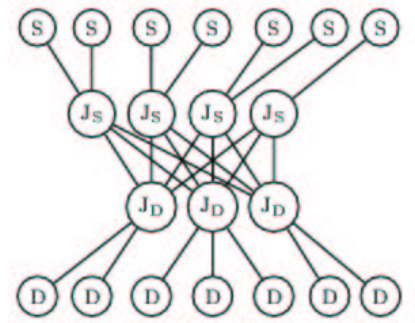

(c)

Figure 2: An illustration of the transformations (left to right) from the original network (a) to the bipartite network (b), and finally, to the network produced by our transformation (c), given two profiles S and D. Nodes labelled with either "S" or "D" belong to the corresponding profile. Nodes labelled with " $\mathrm{J}_{\mathrm{S}}$ " or " $\mathrm{J}_{\mathrm{D}}$ " are junction nodes (see section 4.2).

of the network into a new network such that the concept-to-concept distance is preserved, but without the problem introduced by non-additivity. One possible solution is to construct a complete bipartite graph between the supply nodes and the demand nodes (the nodes in the two context profiles). We set the cost of each edge $(s, d)$ in the bipartite graph to be the concept-to-concept distance between $s$ and $d$ in the original network. Since there is exactly one edge between any pair of nodes, the non-additivity is removed entirely. (See Figures 2(a) and 2(b).) Now, we can apply a network flow solver on the new graph.

However, one problem arises from performing the above mapping - there is a processing bottleneck as a result of the quadratic increase in the number of edges in the new network. Unfortunately, though tractable, polynomial complexity is not always practical. For example, with an average of 900 nodes per profile, making 120 profile comparisons in addition to network re-structuring can take as long as 10 days. ${ }^{2}$ If we choose to use a non-additive distance, the method described above does not scale up well for a large number of comparisons. Next, we present a method to alleviate the complexity issue.

\section{Network Transformation}

One method of alleviating the bottleneck is to reduce the processing load from generating a large number

\footnotetext{
${ }^{2}$ This is tested on a context comparison task not reported in this paper. The code is scripted in perl. The experiment was performed on a machine with two P4 Xeon CPUs running at $3.6 \mathrm{GHz}$, with a $1 \mathrm{MB}$ cache and $6 \mathrm{~GB}$ of memory.
}

of edges. Instead of generating a complete bipartite network, we generate a network which approximates both the structure of the original network as well as that of the complete bipartite network. The goal is to construct a pared-down network such that (a) a reduction in the number of edges improves efficiency, and (b) the resulting distance distortion does not hamper performance significantly.

\subsection{Path Shape in a Hierarchy}

To understand our transformation method, let us further examine the graphical properties of an ontology as a network. In a hierarchical network (e.g., WordNet, Gene Ontology, UMLS), calculating the distance between two concept nodes usually involves travelling "up" and "down" the hierarchy. The simplest route is a single hop from a child to its parent or vice versa. Generally, travelling from one node $i$ to another node $j$ consists of an A-shaped path ascending from node $i$ to a common ancestor of $i$ and $j$, and then descending to node $j$.

Interestingly, our description of the A-shaped path matches the design of a number of concept-toconcept distances. For example, distances that incorporate Resnik's (1995) information content (IC), $-\log (p$ (concept $))$, such as those of Jiang and Conrath (1997) and Lin (1998), consider both the (lowest) common ancestor as well as the two nodes of interest in their calculation.

The complete bipartite graph considered in section 3.2 directly connects each node s in profile $S$ to node $d$ in profile $D$, eliminating the typical Ashaped path in an ontology. This structure solves the 
non-additivity issue, by generating an edge with the exact concept-to-concept distance for each potential node comparison, but, as noted above, is too inefficient. Our solution here is to construct a network that uses the idea of a pared-down A-shaped path to mostly avoid non-additivity, but without the inefficiency of the complete bipartite graph. Thus, as explained in more detail in the following subsections, we trade off the exactness of the distance calculation against the efficiency of the network construction.

\subsection{Network Construction}

In our network construction, we exploit the general notion of an A-shaped path between any two nodes, but replace the "tip" of the A with two nodes. Then for each node $s$ and $d$ in profiles $S$ and $D$, we generate an edge from $\mathrm{s}$ to an ancestor $a_{s}$ of $s$ (the left "branch" of the A), an edge from d to an ancestor $a_{d}$ of $d$ (the right "branch" of the A), and an edge between $a_{s}$ and $a_{d}$ (the two nodes forming the "elongated tip" of the A). Each edge has the exact concept-to-concept distance from the original network, so that the distance between any two nodes $s$ and $d$ is the sum of three exact distances.

The set of ancestor nodes, $a_{s}$ and $a_{d}$, comprise the "junction" points at which the supply from $S$ can be transported across to the nodes in $D$ to satisfy their demand. The set of junction nodes, $J_{P}$, for a profile $P$, must be selected such that for each node $i$ in $P, J_{P}$ contains at least one ancestor of $i$. (See section 4.4 for details on the junction selection process.) The resulting network is constructed by directly connecting each profile to its corresponding junction, then connecting the two junctions in the middle (Figure 2(c)).

The difference between the complete bipartite network and the transformed network here is that, instead of connecting each node in $S$ to every node in $D$, we connect each node in $J_{S}$ to every node in $J_{D}$. Compare the transformed network in Figure 2(c) with the complete bipartite network in Figure 2(b). The complete bipartite component in the transformed network (the middle portion between the junction nodes labelled $J_{S}$ and $J_{D}$ ) is considerably smaller in size. Thus, the number of edges in the transformed network is significantly fewer as well.

Next, we can proceed to define the cost function on the transformed network. Observe that each edge $(s, d)$, with cost $\operatorname{dist}(s, d)$, in the complete bipartite network, where $s \in S, d \in D$, is now instead represented by three edges: $\left(s, a_{s}\right),\left(a_{s}, a_{d}\right)$, and $\left(a_{d}, d\right)$, where $a_{s} \in J_{S}$ and $a_{d} \in J_{D}$. Thus, the transformed distance between $s$ and $d$, dist trans $(s, d)$, becomes:

$\operatorname{dist}_{t r a n s}(s, d)=\operatorname{dist}\left(s, a_{s}\right)+\operatorname{dist}\left(a_{s}, a_{d}\right)+\operatorname{dist}\left(a_{d}, d\right)$

where $\operatorname{dist}(i, j)$ is the precise concept-to-concept distance between $i$ and $j$ in the original network. Once we have set up the transformed network, we can solve the MCF in this network, yielding the distance between the two (supply and demand) profiles.

\subsection{Distance Distortion}

Because the distance between nodes $s$ and $d$ is now calculated as the sum of three distances (eqn. (6)), some distortion may result for non-additive conceptto-concept distances. To illustrate the distortion effect, consider Jiang and Conrath's (1997) distance:

$$
\operatorname{dist}_{j c}(i, j)=I C(i)+I C(j)-2 I C(L C S(i, j))
$$

where $I C(i)$ is the information content of a node $i$, and $L C S(i, j)$ is the lowest common subsumer of nodes $i$ and $j$. This distance measures the difference in information content between the concepts and their lowest common subsumers.

After the transformation, the distance is distorted in the following way. If $i$ and $j$ have no common junction ancestor, then $\operatorname{dist}_{j c_{\text {trans }}}(i, j)$ becomes:

$$
\begin{aligned}
\operatorname{dist}_{j c_{\text {trans }}}(i, j)= & {\left[I C(i)+I C\left(a_{i}\right)-2 I C\left(a_{i}\right)\right]+} \\
& {\left[I C(j)+I C\left(a_{j}\right)-2 I C\left(a_{j}\right)\right]+} \\
& {\left[I C\left(a_{i}\right)+I C\left(a_{j}\right)-2 I C\left(L C S\left(a_{i}, a_{j}\right)\right)\right] } \\
= & I C(i)+\operatorname{IC}(j)-2 I C\left(L C S\left(a_{i}, a_{j}\right)\right)(8)
\end{aligned}
$$

where $a_{i}$ and $a_{j}$ are the junction ancestors of $i$ and $j$, respectively. Otherwise, if $i$ and $j$ share a common ancestor $a$ at the junction, then $d_{i s t_{j c_{\text {trans }}}}(i, j)$ becomes $I C(i)+I C(j)-2 I C(a)$, where the term $2 \operatorname{IC}\left(\operatorname{LCS}\left(a_{i}, a_{j}\right)\right)$ in eqn. (8) is replaced by $2 I C(a)$. In either case, the transformation replaces the lowest common subsumer $\operatorname{LCS}(i, j)$ in eqn. (7) with some other common subsumer $C S(i, j)\left(L C S\left(a_{i}, a_{j}\right)\right.$ or $a$, mentioned above). Unless $C S(i, j)=L C S(i, j)$, the distance is distorted by using a less precise quantity, $\operatorname{IC}(C S(i, j))$.

Note that the information content of a concept is given by its maximum likelihood estimate based on 
its frequency in a large corpus. An increment in the frequency of a concept leads to an increment in the frequency of all its ancestors. Due to the frequency percolation, concepts with a small depth tend to accumulate higher counts than those deeper in the hierarchy (note the difference in depth: depth ${ }_{C S(i, j)}$ $\left.\leq d e p t h_{L C S(i, j)}\right)$. Thus, we expect the information content of a concept to be higher than its ancestors, i.e., a concept is more semantically specific than its ancestors, which is captured by the use of the negative $\log$ function in the definition of IC. The transformed distance is distorted accordingly $(I C(C S(i, j)) \leq \operatorname{IC}(L C S(i, j)))$.

\subsection{Junction Selection}

Selection of junction nodes is a key component of the network transformation. Trivially, a junction consisting of profile nodes yields a network equivalent to the complete bipartite network. The key is to select a junction that is considerably smaller in size than its corresponding profile, hence, cutting down the number of edges generated, which results in significant savings in complexity.

Note that there is a tradeoff between the overall computational efficiency and the similarity between the transformed network and the complete bipartite network. The closer the junctions are to the corresponding profiles, the closer the transformed network resembles the complete bipartite network. Though the distance calculation is more accurate, such a network is also more expensive to process. On the other hand, there are fewer nodes in a junction as it approaches the root level, but there is more distortion in the transformed concept-to-concept distance. Clearly, it is important to balance the two factors.

Selecting junction nodes involves finding a smaller set of ancestor nodes representing the profile nodes in a hierarchy. In other words, the junction can be viewed as an alternative representation which is a generalization of the profile nodes. In addition to the profile nodes, the junction nodes are also included in the transformed network. They may provide extra information about the corresponding context.

Finding a generalization of a profile is explored in the works of Clark and Weir (2002) and Li and Abe (1998). Unfortunately, the complexity of these algo- rithms is quadratic (the former) or cubic (the latter) in the number of nodes in a network, which is unacceptably expensive for our transformation method. Note that to ensure every profile node has an ancestor node in the junction, the selection process has a linear lower bound. To keep the cost low, it is best to keep a linear complexity for the junction selection process. However, if this is not possible, it should be significantly less expensive than a quadratic complexity. We will empirically explore the process further in section 5.3.

\section{Context Comparison}

As alluded to earlier, our network flow method provides an alternative to a purely distributional and non-graphical approach to context comparison. In this paper, we will test both variants of our method (with or without the transformation in section 4) in a name disambiguation task in which the context words within a small window surrounding the ambiguous words are compared. Our preliminary analysis shows that our general network flow framework is robust and efficient.

\subsection{Name Disambiguation}

The goal for name disambiguation is to classify each ambiguous instance on the basis of its surrounding context. One approach is to use an unsupervised method such as clustering. This involves making a large number of pairwise comparisons between individual contexts. Given that there is an overhead to incorporating ontological information, our network flow method does not compute distances as efficiently as calculating a purely arithmetic distance such as cosine or Euclidean distance. Our alternative approach is to use minimal training data. Using a handful of contexts, we can build a "gold standard" profile for each sense of an ambiguous name by using the context words of a small number of instances. We then compare the context profile of each instance to the gold standards. Each instance is given the label of the gold standard profile to which its context profile is the closest.

\subsection{Experimental Setup}

In our name disambiguation experiment, we use the data collected by Pedersen et al. (2005) for their name discrimination task. This data is taken from 


\begin{tabular}{|l|c|cc|cc|}
\hline Name Pairs & Baseline & 200 (Full) & 200 (Trans) & 100 (Full) & 100 (Trans) \\
\hline Ronaldo/David Beckham & 0.69 & 0.80 & 0.88 & 0.79 & 0.84 \\
Tajik/Rolf Ekeus & 0.74 & 0.97 & 0.99 & 0.98 & 0.99 \\
Microsoft/IBM & 0.59 & 0.73 & 0.75 & 0.73 & 0.71 \\
Shimon Peres/Slobodan Milosevic & 0.56 & 0.96 & 0.99 & 0.97 & 0.99 \\
Jordan/Egyptian & 0.54 & 0.77 & 0.76 & 0.74 & 0.76 \\
Japan/France & 0.51 & 0.75 & 0.82 & 0.75 & 0.83 \\
\hline Weighted Average & 0.53 & 0.77 & 0.82 & 0.76 & 0.82 \\
\hline
\end{tabular}

Table 1: Name disambiguation results (accuracy/F-measure) at a glance. The baseline is the relative frequency of the majority name. "200" and "100" give the averaged results (over five different runs) using 200 and 100 randomly selected training instances per ambiguous name. The weighted average is calculated based on the number of test instances per task. "Full" and "Trans" refer to the results using the full network (pre-transformation) or the pared-down network (with transformation), respectively.

the Agence France Press English Service portion of the GigaWord English corpus distributed by the Linguistic Data Consortium. It consists of the contexts of six pairs of names, including: the names of two soccer players (Ronaldo and David Beckham); an ethnic group and a diplomat (Tajik and Rolf Ekeus); two companies (Microsoft and IBM); two politicians (Shimon Peres and Slobodan Milosevic); a nation and a nationality (Jordan and Egyptian); and two countries (France and Japan). These name pairs are selected by Pedersen et al. (2005) to reflect a range of confusability between names.

Each pair of names serves as one of six name disambiguation tasks. Each name instance consists of a context window of 50 words ( 25 words to the left and to the right of the target name), with the target name obfuscated. For example, for the task of distinguishing "David Beckham" and "Ronaldo", the target name in each instance becomes "David_BeckhamRonaldo". The goal is to recover the correct target name in each instance.

\subsection{Junction Selection}

We reported earlier that a complete bipartite graph with 900 nodes is too expensive to process. Our first attempt is to select a junction on the basis of the number of nodes it contains. Here, the junctions we select are simple to find by taking a top-down approach. We start at the top nine root nodes of WordNet (nodes of zero depth) and proceed downwards. We limit the search within the top two levels because the second level consists of 158 nodes, while the following level consists of 1307 nodes, which, clearly, exceeds 900 nodes. Here, we select the junction which consists of eight of the top root nodes (silbings of entity) and the children of entity, given that entity is semantically more general than its siblings. ${ }^{3}$

In our current experiment, we use Jiang and Conrath's distance for its ease of analysis. As shown in section 4.3, only one term in the distance, $\operatorname{IC}(\operatorname{LCS}(i, j))$, is replaced because of the use of the junction nodes. Any change in the performance (in comparison to our method without the transformation) can be attributed to the distance distortion as a result of this term being replaced. The analysis of experimental results (next section) is made easy because we can assess the goodness of the transformation given the selected junction-a significant degradation in performance is an indication that the junction nodes should be brought closer to the profile nodes, yielding a more precise distance.

\section{Results and Analysis}

To compare the two variants of our method, we perform our name disambiguation experiment using 100 and 200 training instances per ambiguous name to create the gold standard profiles. See Table 1 for the results. Comparing the results using the full network and the transformed network, observe that there is very little performance degradation; in fact, in most cases, there is an increase in accuracy (the difference is significant, paired t-test with $p \ll 0.05)$.

Distance Transformation In Jiang and Conrath's formulation, the network transformation replaces the term $2 I C(L C S(i, j))$ with $2 I C(C S(i, j))$, where $C S(i, j)$ is some common ancestor of $i$ and

\footnotetext{
${ }^{3}$ Note that the complexity of this selection process is linear, since all profile nodes must be examined to ensure they have an ancestor in the junction; any profile node of which no junction node is an ancestor is added to the junction. This process can only be avoided by using junction nodes of zero depth exclusively.
} 
$j$, whose depth is small. Junction nodes with a small depth distort the distance more than those with a larger depth. Surprisingly, our experiment indicates that using such nodes produces equally good or better performance. This suggests that selecting a junction with a larger depth, at least for the data in this task, is not necessary.

Speed Improvement In comparison to our reported running time on the pre-transformation network (120 comparisons running for 10 days), on the same machine, making 12,000 comparisons can now be accomplished within two hours. In terms of complexity, if we have $n$ profile nodes and $j$ junction nodes, the number of edges to be processed is $O\left(n+j^{2}\right)$. Given that our junctions have significantly fewer nodes than the original profiles, the running time is significantly less than quadratic in the number of profile nodes.

\section{Conclusions}

We have given an overview of our network flow formalism which seamlessly combines distributional and ontological information. Given a suitable ontology, a context vector of word frequencies can be transformed into a context profile-a frequency distribution over the concepts in the ontology. In contrast to traditional non-graphical approaches to measuring only the distributional distance between context vectors, we provide a graphical formalism which incorporates both the semantic distance of the component nodes as well as the distributional differences between the context profiles. By taking advantage of the graphical structure of an ontology, our method allows a systematic and meaningful way of abstracting over words in a context, and by extension, a meaningful way of comparing contexts.

One concern with our method in its pretransformation form is its inability to incorporate sophisticated concept-to-concept semantic distances efficiently. To remedy this, we propose a novel technique that mimics the structure of the more computationally intensive network. Our preliminary evaluation shows that the transformation does not hamper the method's ability to make fine-grained semantic distinctions, and the computational complexity is drastically reduced as well. Generally, our network flow method presents a highly competitive alterna- tive to a purely distributional and non-graphical approach.

In our on-going work, we are further exploring how the choice of junction influences the performance of different types of concept-to-concept semantic distances. For example, would a bottom-up junction selection approach (from the profile nodes instead of from the root level) result in better performance? In addition, we intend to examine the graphical properties of the individual profiles as well as the routes between the concepts across profiles selected by our network flow methods. Such analyses will help us gain insight into the strengths (and weaknesses) of taking advantage of a graphical representation of contexts as well as treating an ontology as a metric space for context comparisons.

\section{References}

Budanitsky, A. and Hirst, G. (2006). Evaluating WordNet-based measures of semantic distance. Computational Linguistics. To appear.

Clark, S. and Weir, D. (2002). Class-based probability estimation using a semantic hierarchy. Computational Linguistics, 28(2):187-206.

Jiang, J. and Conrath, D. (1997). Semantic similarity based on corpus statistics and lexical taxonomy. In Proceedings on the International Conference on Research in Computational Linguistics, pages 19-33.

Li, H. and Abe, N. (1998). Word clustering and disambiguation based on co-occurrence data. In Proceedings of COLINGACL 1998, pages 749-755.

Lin, D. (1998). An information-theoretic definition of similarity. In Proceedings of International Conference on Machine Learning.

Mihalcea, R. (2006). Random walks on text structures. In Proceedings of CICLing 2006, pages 249-262.

Miller, G. A. and Charles, W. G. (1991). Contextual correlates of semantic similarity. Language and Cognitive Processes, 6(1):1-28.

Navigli, R. and Velardi, P. (2005). Structural semantic interconnections: A knowledge-based approach to word sense disambiguation. IEEE Transactions on Pattern Analysis and Machine Intelligence, 27(7).

Pedersen, T., Purandare, A., and Kulkarni, A. (2005). Name discrimination by clustering similar context. In Proceedings of the Sixth International Conference on Intelligent Text Processing and Computational Linguistics.

Resnik, P. (1995). Using information content to evaluate semantic similarity in a taxonomy. In Proceedings of the 14th International Joint Conference on Artificial Intelligence.

Schütze, H. (1998). Automatic word sense discrimination. Computational Linguistics, 24(1):97-123.

Xu, W., Liu, X., and Gong, Y. (2003). Document clustering based on non-negative matrix factorization. In Proceedings of the 26th ACM SIGIR Conference. 